\title{
Desarrollo de una guía de campo para el avistamiento de delfines en Puerto El Morro (Ecuador)
}

\author{
Ricardo Villalba Briones* Maria Vanesa Sanchez Crow** \\ Kael Andre Sellan Moncayo**** \\ Escuela Superior Politécnica del Litoral (Ecuador)
}

\begin{abstract}
Resumen: Las guías de campo para avistamientos de cetáceos son herramientas didácticas que ayudan a cumplir con los objetivos de las actividades ecoturísticas favoreciendo la preparación de los guías turísticos. En este trabajo se examina la importancia del ecoturismo de delfines y se presenta un método para ajustar las guías de campo a las características locales, en este caso, las de Puerto El Morro (Ecuador). Proporcionando a los guías información que fomente la valoración y conservación de los bufeos o delfines nariz de botella Tursiops truncatus, se potencia el conocimiento en el enlace principal entre el público y los delfines; es decir, los guías de las actividades de avistamiento de delfines. Se utilizaron cuestionarios realizados a usuarios para determinar los atractivos de mayor interés del Refugio de Vida Silvestre Manglares del Morro en el turista y se evaluó la fortaleza de la guianza en las temáticas ecológicas relacionadas. El $76 \%$ de los usuarios de las operadoras encuestados ( $\mathrm{N}=22$ ) percibieron al delfín como atractivo turístico principal, sobre las aves y el ecosistema de manglar. Estos elementos eran cubiertos de forma poco desarrollada durante la guianza por lo que se justificó incluirlos estratégicamente en la guía de campo para el avistamiento de delfines.
\end{abstract}

Palabras Clave: Delfin; Guía; Conservación; Ecoturismo; Diseño; Manual.

Design of a field guide for the sighting of dolphins Tursiops truncatus in Puerto El Morro (Ecuador).

Abstract: The instructional guides for whale watching activities are didactic tools that support achieving the objectives of ecotourism, favoring the preparation of tourist guides. Providing guides with information that encourages the valuation and conservation of bottlenose dolphins Tursiops truncatus, locally known as "bufeos", the printed material strengthens the capabilities of the main link between the public and the dolphins; the tour guides of dolphin watching activities. Surveys of the clients and guides were conducted to determine the topics of the greatest interest to the tourist of the Mangrove Wildlife Refuge of El Morro and tourist guides were evaluated on guidance quality over related ecological topics. $76 \%$ of the users of the operators surveyed $(\mathrm{N}=22)$ perceived the dolphin as the main tourist attraction against avian species and the mangrove ecosystem. The identified topics were selected to develop the handbook on Dolphin watching in Puerto El Morro.

Keywords: Dolphin; Guide; Conservation; Ecotourism; Design; Handbook.

\section{Introducción}

La atracción que generan los delfines ha sido constatada desde la época antigua, tanto para los romanos como para los griegos de la época clásica, el delfín era visto como un amigo del hombre, que salvaba a marineros del ahogamiento, ayudaba a llevar peces a la red. El delfín era coronado como el rey del mar, e incluso, entre los textos clásicos, se registran relatos de amistad y de amor entre humanos y delfines, agregándole, en variadas ocasiones, una naturaleza divina (Hicks, 1887; Alpers, 1961; Traina, 2005). En la mitología ancestral de los Kunas de Panamá en cambio el delfín es quien trae a los recién nacidos (Mauri, 2004). También en la época medieval se le enaltecía como un ser de naturaleza cariñosa

* Escuela Superior Politécnica del Litoral (Ecuador); E-mail: rvillalba@espol.edu.ec ; https://orcid.org/0000-0002-5577-1705

** Escuela Superior Politécnica del Litoral (Ecuador); E-mail: Mavasanc@espol.edu.ec

*** Escuela Superior Politécnica del Litoral (Ecuador); E-mail: kaansell@espol.edu.ec 
en los bestiarios europeos como en "La leyenda del delfín enamorado" o de socorredor en "The tale of the helpful dolphin" (Deyermond, 2004). En el conocimiento ancestral de Ecuador se cuenta que en el río Napo el delfín de río acudía en forma humana a fiestas para cortejar a las muchachas que encantaba y dejaba embarazadas (Tejedor, 2016).

La ciencia de la biología ha avanzado en muchos campos relacionados a los estudios de la inteligencia animal otorgando a unos pocos, entre ellos a los delfines, la capacidad de comportamiento moral o justicia (Bekoff, 2004). Tras tantas investigaciones sobre delfines se ha constatado su alta capacidad a la hora resolver problemas, su desarrollado lenguaje, sus relaciones sociales complejas y duraderas, y sobre todo la conciencia de sí mismos que poseen, es decir el hecho de que se reconozcan a sí mismos (Marten y Psarakos, 1994; Reiss y Marino, 2001). El aumento de conocimiento en el área ha suscitado que en el congreso de 2010 de la mayor organización científica del mundo en términos de afiliados, la Asociación Estadounidense para el Avance de la Ciencia Americana (American Association for the Advancement of Science o AAAS), se expusiera que los delfines debían tener incluso tantos derechos como los humanos debido a su inteligencia (Grimm y Miller, 2010; BBC press, 2012).

La presencia del delfín genera una atracción que hoy en día se demuestra a través de la gran cantidad de usuarios que tiene el turismo de cetáceos, atrayendo ingresos en transporte, restaurantes y demás servicios, además del avistamiento y guianza hasta ellos. De hecho, esta actividad turística genera cada vez más ingresos en América Latina, y su crecimiento ha sido del 17.8\% anual en la Republica del Ecuador en el lapso desde 1991 hasta 2006 (Hoyt y Iñiguez, 2008).

La observación de cetáceos se ha convertido en una fuente importante de ingresos para comunidades como la de Puerto López y Puerto El Morro, regulada por normativas destinadas a proteger este valor natural como un recurso (Ministerio de Turismo, 2014), siendo explotada no sosteniblemente cuando la normativa no es respetada, lo cual pone en riesgo la permanencia de la población de delfines en el área. Cabe destacar que los botes privados, de pesca y comerciales ajenos al ecoturismo pueden incrementar el impacto por medio de diferentes acciones (Spalding y Blumenfeld, 1997), como por ejemplo al pasar a velocidad media o alta sobre el grupo de delfines, generando segregación y huida en los individuos, afectando, al mismo tiempo, a la actividad turística debido a que también se interrumpe inexorablemente el avistamiento.

\section{Figura 1: Bufeo o delfín nariz de botella, Tursiops truncatus en el Refugio de Vida Silvestre Manglares del Morro.}

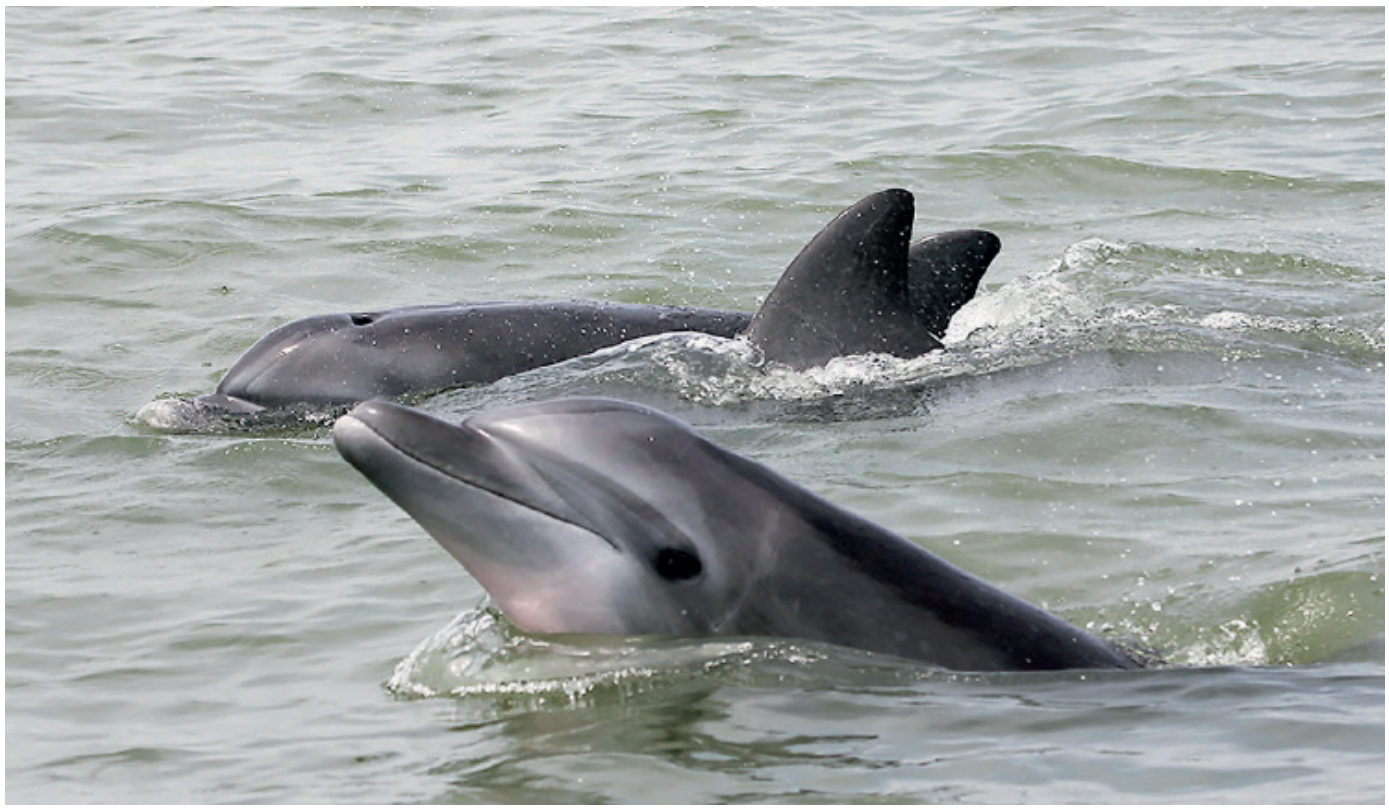

Foto: Fernando Félix. 
La investigación para el desarrollo de la guía de campo para avistamiento de delfines (véase figura 1) se llevó a cabo en Puerto El Morro, recinto de la parroquia El Morro, a 90 kilómetros de guayaquil (Ecuador) (Sistema Nacional de Información, 2015), donde se encuentran las operadoras turísticas que organizan actividades de avistamientos de delfines dirigidas al contiguo Refugio de Vida Silvestre Manglares El Morro (REVISMEM), área protegida de ecosistema de manglar con una extensión de 10.030 hectáreas, con itinerarios que en ocasiones llegan hasta las aguas del puerto de la localidad adyacente, Posorja (véase mapa 1). Los itinerarios ecoturísticos de Puerto El Morro ofertan un paseo en bote con atractivos naturales reconocidos para la observación de la naturaleza del REVISMEM, su ecosistema de manglar, las aves que habitan (Pelegrín, 2014) y la observación de delfines. La comunidad de Puerto El Morro se encuentra en la localidad de El Morro, la cual consta de 5.210 habitantes y un nivel administrativo de gobierno autónomo descentralizado de parroquia que se caracteriza por sus actividades turísticas, agrícolas, pesqueras y ganaderas (Sistema Nacional de información, 2015; Aguirre-Torres, 2019).

Mapa 1: Localización de Puerto El Morro y Posorja evidenciando las localizaciones de los delfines durante las actividades ecoturísticas.

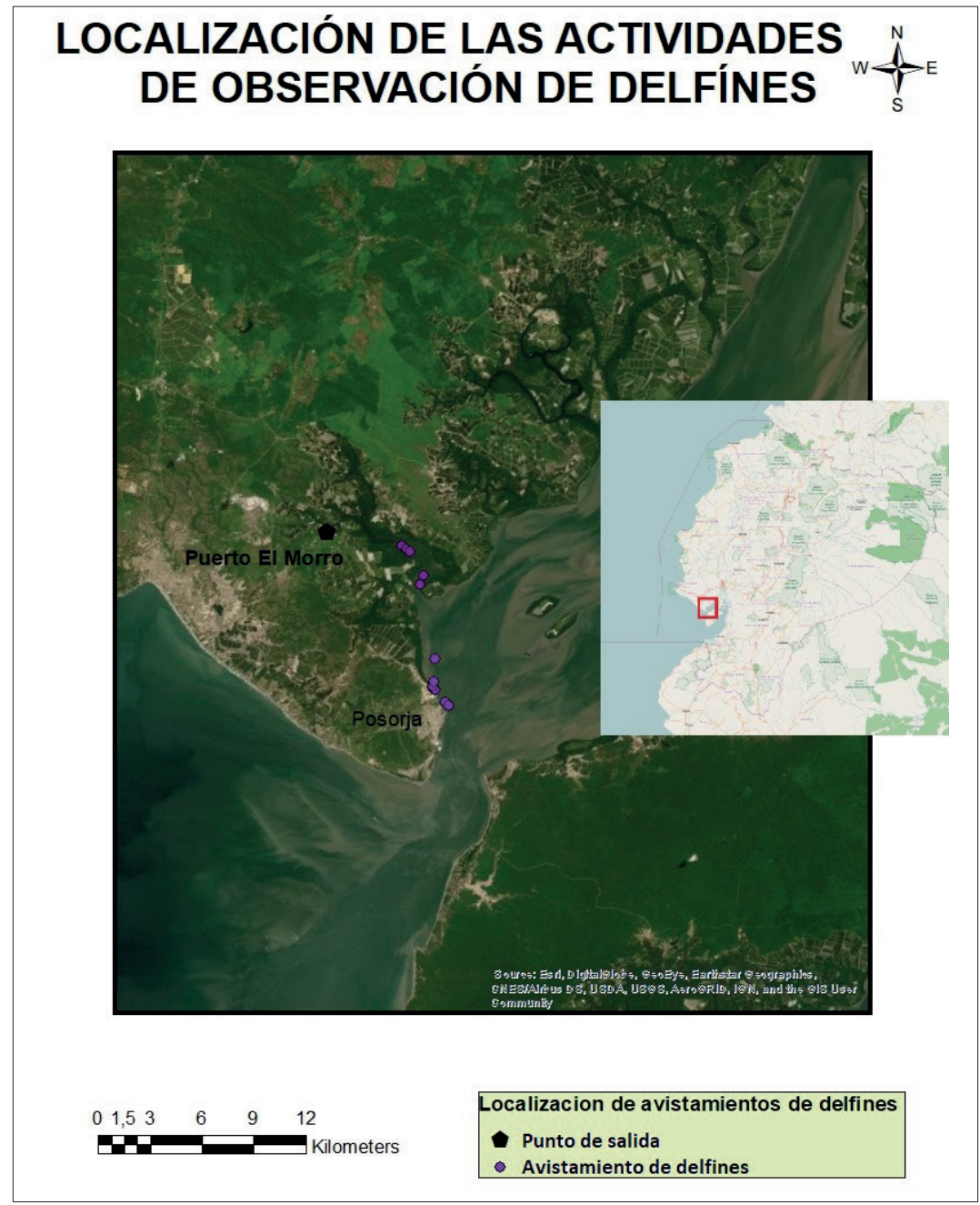

Fuente: Elaboración propia (software ESRI, Arcgis 10.3). 
La experiencia de avistar mamíferos marinos en la naturaleza contribuye adoptar actitudes proambientales y concientización a guía turísticos y usuarios del servicio ecoturístico (Walker et al., 2015). El conocimiento de las características biológicas, fisiológicas y perceptivas de los delfines (delfines nariz de botella Tursiops truncatus en el caso del REVISMEM, también llamado bufeo en la localidad de Puerto El Morro), posibilita una mayor eficiencia a la hora de protegerlos mientras se esté dando la actividad turística de avistamiento de delfines, (Daniel et al., 2002; García-Cegarra y Pacheco, 2017), ya que facilita la comprensión de la razón de ser de las normativas al respecto y, por ende, su respeto. Debido a ello, el conseguir un manuscrito que permita favorecer el aprendizaje autónomo de ellos guías, que potencie la conservación y este localmente adaptado, puede constituir un recurso que influya positivamente de forma continuada. Teniendo en cuenta las amenazas que sufren los delfines y su importancia tanto ecológica como cultural, una guía de campo puede ser un recurso de apoyo que enriquezca directamente la actividad ecoturística y la eficiencia del guía en su labor. En este trabajo se presenta el estudio previo a la realización de una guía de campo para el avistamiento de bufeos Tursiops truncatus en Puerto El Morro (Ecuador), identificando las preferencias sobre los atractivos turísticos más populares para los usuarios durante los avistamientos de delfines, registrando percepciones sobre la normativa de avistamiento de cetáceos y evaluando el contenido informacional ofrecido por los guías ecoturísticos. Para conseguir estos objetivos se utilizó la herramienta de medición de cuestionario tanto con preguntas abiertas como cerradas para usuarios y un formulario para guías, que luego fueron analizados cuantitativamente mediante el software R-commander (Fox, Bouchet-Valat, 2019).

\section{Amenazas}

Los bufeos del Golfo de Guayaquil son depredadores que se encuentran en la cima de la cadena trófica (Jiménez y Álava, 2015). En otros lugares, orcas y grandes tiburones como el tiburón tigre depredan delfines, sobre todo a las crías, por lo que los delfines los evitan (Ballance, 2016) pero no se ha reportado interacciones de estas especies en el Golfo de Guayaquil (Félix 2013). En los delfines el grupo favorece tanto en la obtención de alimento como en la protección de los individuos que lo componen. Para su protección desarrollan actividades cooperativas complejas de protección, como rodeando a las crías, buscando la confusión del depredador y protección física mientras otros individuos se turnan amenazantes sobre los tiburones (Ballance, 2016). Las poblaciones de delfines mueren debido a varias causas, pero cabe destacar que en la comunidad de delfines de Puerto El Morro y Posorja tienen un alto grado de marcas e historial de colisiones o golpes de embarcaciones (véase figura 2) y se enredan en redes de pesca artesanales que en ocasiones generan su muerte (Félix, 2013), por otra parte, en general, en delfines, las principales amenazas suelen ser la falta de presas por excesiva presión pesquera, los pesticidas y la polución del agua que afectan a su salud y reproducción, los parásitos y las enfermedades víricas, y el tráfico de embarcaciones tanto por contaminación acústica como por colisiones (Stensland et al., 2006; Currey et al., 2012), además de otros depredadores mayores (Balance, 2016).

\section{Figura 2: Imagen de delfín con señales de impactos en el Refugio de Vida Silvestre Manglares del Morro.}

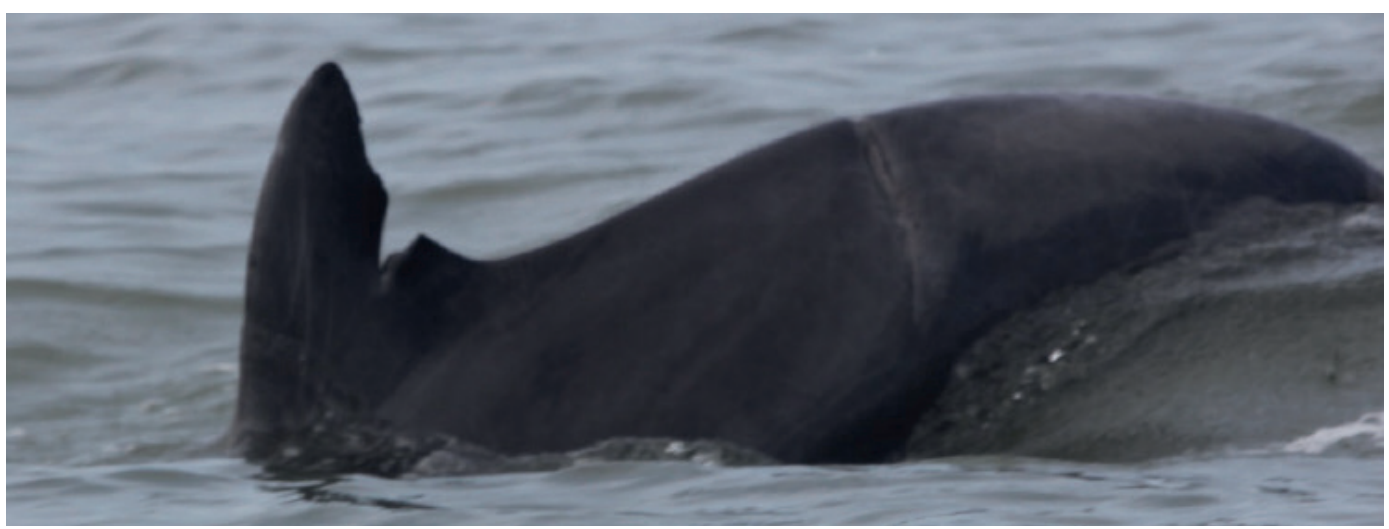

Foto: Fernando Félix. 


\section{Importancia ecológica de las poblaciones de delfines}

Como depredador de la cima de la cadena trófica, los delfines (véase figura 3) estabilizan las poblaciones de especies que consumen (peces mayormente) y su sola presencia hace que los peces no consuman en exceso otras especies de plantas o animales.

\section{Figura 3: Bufeo Tursiops truncatus del Refugio de Vida Silvestre Manglares del Morro.}

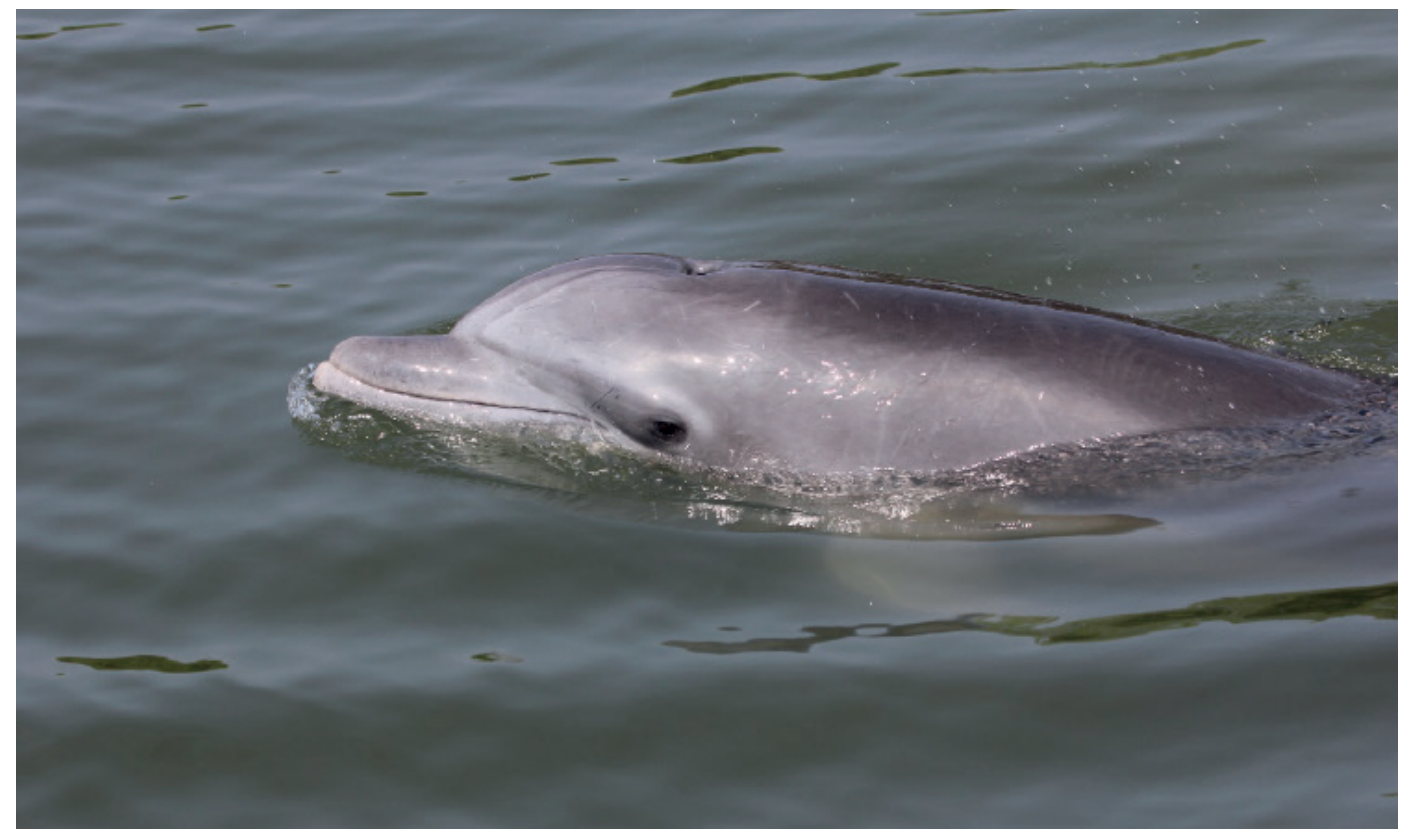

Foto: Fernando Félix.

La presencia de los delfines provoca que los peces, que son sus presas, no afecten en exceso los recursos de forma que no se desgasten las posibles fuentes de alimento manteniendo el equilibrio del ecosistema (Dill, Heithaus y Walters, 2003). En el Mar Negro, el hecho de que hubiera menos delfines y otros grandes depredadores que se alimentaban de peces, en la década 1970, hizo que los pequeños peces que consumían plancton (pequeños animales como larvas, las larvas de cangrejos que flotan en el agua) abundaran, lo que hizo que el plancton disminuyera y que fitoplancton (pequeñas plantas microscópicas que dan al agua un color verde) se duplicara (Daniel et al., 2002; Daskalov et al., 2007). Tras ello las medusas o animales gelatinosos se incrementaron. La cadena trófica se vio perturbada, se dañó, y cambió la abundancia de animales. Estos estudios apuntan a que la presencia del delfín como depredador en la cadena trófica favorece al equilibrio del ecosistema y que desaparezca afecta al a las poblaciones de peces, influenciado indirectamente a los demás seres vivos en forma de cascada (Akoglu et al., 2014).

Además, el llamado 'efecto riesgo' induce cambios en el comportamiento de las presas, en este caso, los peces. La presencia del depredador hace que no se alimenten demasiado de su alimento preferido en donde este se encuentra, ya que se exponen a los depredadores, (Baum y Worm, 2008).

Estudios sobre los sonidos producidos por cetáceos, demuestran que su presencia influencia a los peces cambiando su comportamiento adquiriendo una actitud de defensa para evitar la depredación (Remage-Healey, Nowacek y Bass, 2006; Wilson y Dill, 2002). Esto hace que los consumidores, los peces, por ejemplo, no agoten el recurso de su alimento preferido por que, si ocurriera, afectaría también notablemente al ecosistema en forma de cascada o cadena. La abundancia de grandes depredadores está relacionada con el equilibrio del ecosistema que favorece la prevalencia a largo plazo de los recursos que los humanos disfrutan (Frank et al., 2005; Casini et al., 2008). 


\section{Figura 4: Bufeo, delfín nariz de botella, Tursiops truncatus acercándose a bote con motor en neutro.}

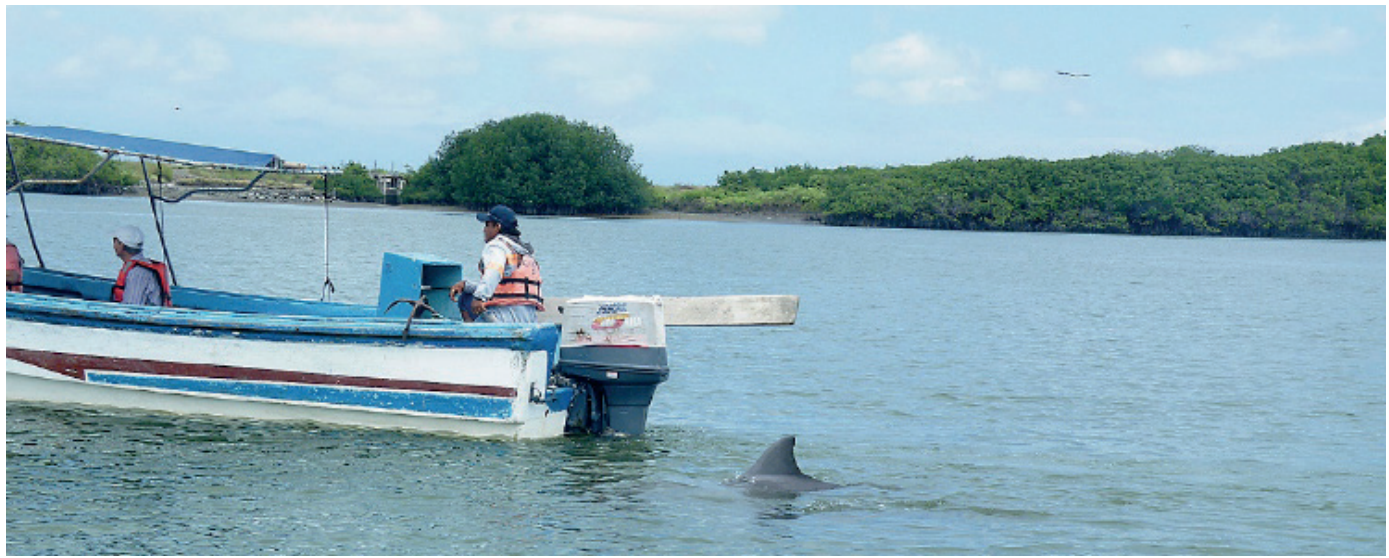

Foto: Eduardo Rafael Correa.

\section{Efectos de embarcaciones en delfines}

Los bufeos cambian de dirección cuando hay botes en su itinerario, situación que la normativa prohíbe, bucean para evitar a los barcos y huyen cuando se sienten acosados afectando a sus actividades normales como la alimentación o socialización. El ruido los aleja y escapan de lo que les estresa o molesta cuando tienen ocasión, o pueden permitírselo, y como conductores debemos evitar esas conductas. El ruido por embarcaciones comerciales o turísticas generan un sonido que hace que la comunicación entre delfines y su ecolocación se vean perjudicadas y puede afectar a el número de delfines en el área (Nakahara, 1999). Según el estudio de 6 años en Irlanda Culloch (2016) el ruido aleja a los cetáceos, se estudiaron a los delfines comunes, rorcuales comunes y marsopas (respectivamente Delphinus delphis, Balaenoptera acutorostrata, Phocoena phocoena) y su presencia en la zona disminuyó por obras y tráfico de embarcaciones. Como la mayoría de los cetáceos dependen fuertemente del sonido para comunicarse, cazar y navegar (Culloch et al., 2016), el sonido ambiental afecta a las actividades de los delfines por lo que las normativas sobre avistamientos de cetáceos (bufeos) tratan de mitigar, disminuir, el impacto de las embarcaciones cerca de los delfines, obligando a los boteros a bajar la velocidad y en el caso de Ecuador a no acercarse a menos de 50 metros del delfín (Acuerdo Ministerial 4. Registro Oficial 278, 2014). Los delfines responden de diferentes formas ante la presencia de embarcaciones que pueden afectar a su conservación a largo plazo (Nowacek, Wells y Solow, 2001; Hastie et al., 2003).

Entre ellos se han descrito cambios en su patrón de movimiento (Hastie, 2003), buceos durante mayor tiempo y trayecto (Janik y Thompson, 1996; Nowacek et al, 2001) e incluso alteraciones en la selección de hábitat a la hora de alimentarse (Allen y Read, 2000).

\section{Normativas}

Los avistamientos de cetáceos en forma de turismo se dieron por primera vez en 1950 (Forestell, 2007), en Latinoamérica estas actividades existen desde 1970, posteriormente, la observación de ballenas se convirtió en un negocio anual de turismo de mil millones de dólares empleando a miles de trabajadores y sirviendo a millones de observadores de ballenas cada año (Hoyt y Iñíguez, 2008).

Varias preocupaciones han surgido desde entonces, incluyendo la preocupación por la influencia de los barcos, por la tecnología utilizada en el medio acuático y por las actividades de humanas, que perturban el hábitat natural de las ballenas y delfines, poniéndolos en peligro e interrumpiendo sus patrones de cría y migración naturales, lo cual, podría poner en peligro a las especies. En 1993, la Comisión Ballenera Internacional realizó una evaluación de las actividades de observación de ballenas y en 1996 adoptó una resolución y estableció unas directrices generales para la observación de cetáceos que recopilaron regulaciones de observación de ballenas de todo el mundo para garantizar la seguridad de las ballenas y para seguir siendo una actividad recreativa para los turistas. En la figura 5 vemos la versión australiana y 
su similitud con la establecida según la legislación ecuatoriana, similitud, que demuestra que el consenso internacional referente a la protección de las especies es aceptado internacionalmente y está cimentada sobre bases sólidas de investigación, que deben aplicarse siempre para el avistamiento de delfines. Entre las regulaciones más importantes para el bienestar de los bufeos revisados durante el proyecto de la ESPOL 'Fortalecimiento de las capacidades de avistamiento y sensibilización hacia los bufeos y los valores de la naturaleza por los operadores de turismo en el área de Puerto El Morro' se encuentran las siguientes: los 50 metros de distancia a el delfín como máximo (mayor distancia en caso de que haya crías), el número de tres embarcaciones por avistamiento (debiendo esperar en caso de ser más a que se retiren), la baja velocidad de la embarcación al acercarse a los delfines, la prohibición de acosar o perseguir a los delfines, no interrumpir el itinerario de los delfines colocándose a los costados y el posicionamiento en neutro del motor en zona de avistamiento entre otros. Para más información al respecto de la "Normativa que regula observación de ballenas y delfines en el Ecuador" (Ministerio de Turismo, 2014) véase link en el apartado referencias. En el caso del Golfo de Guayaquil se recomienda que la comunidad pesquera sea consciente del impacto que causa invadir la zona de actividad de los bufeos, ya que todo tráfico de embarcaciones puede afectar negativamente al bienestar y estancia de la población de delfines en el lugar.

Figura 5: Actividad de guianza en salida turística.

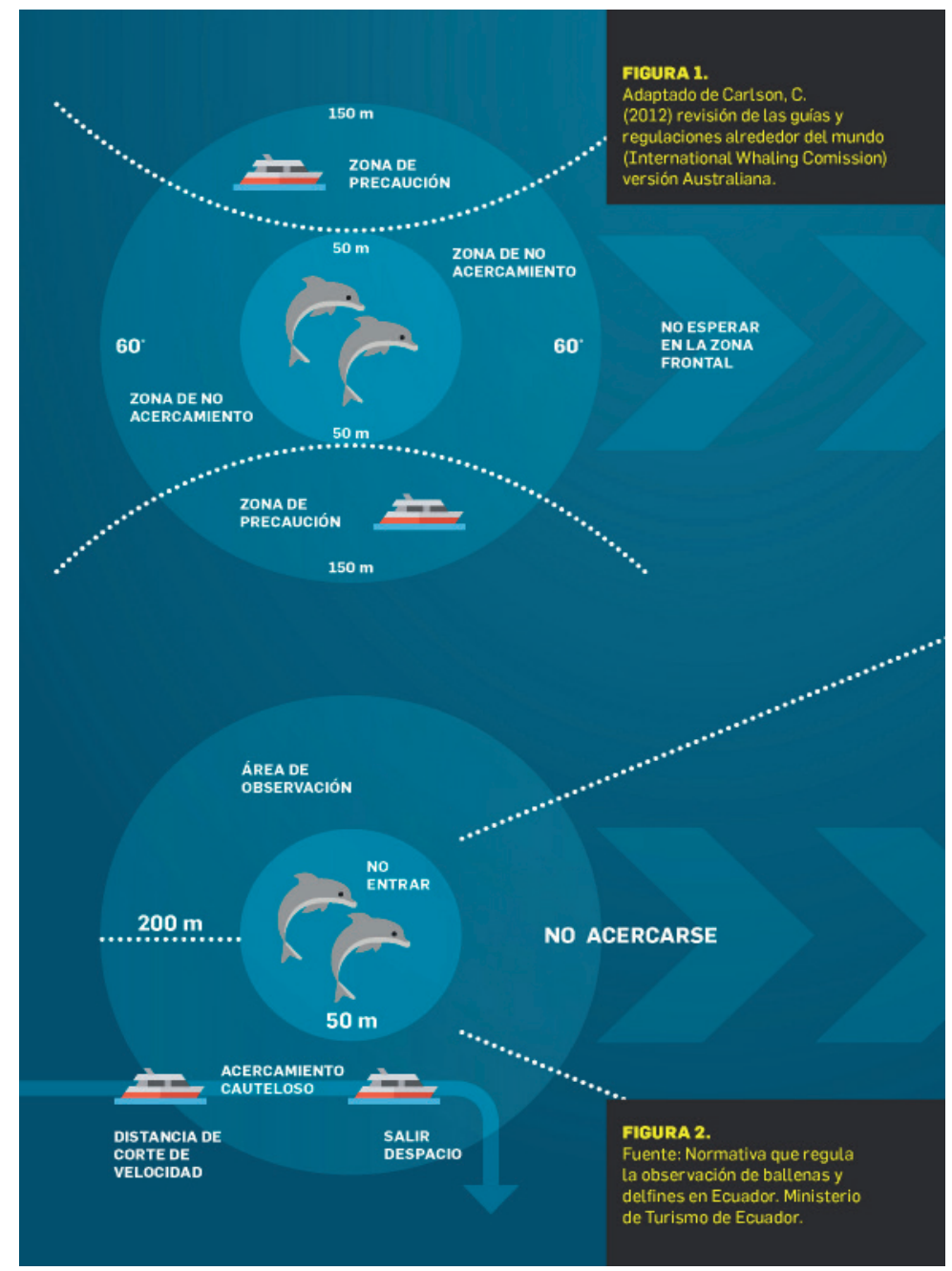

Fuente: Rafael Correa 


\section{El valor de la observación de cetáceos y la importancia del guía}

Probablemente, el valor más importante que pueda tener esta actividad es el potencial para educar a las personas de todas las edades para apreciar, valorar y comprender a los mamíferos marinos (IFAW, 1997)

El Fondo Internacional para el Bienestar Animal (IFAW, por sus siglas en inglés) propone siete valores educativos del avistamiento de cetáceos:

1) Los cetáceos son emblemas para promover la toma de conciencia sobre especies en peligro de extinción y protección del hábitat.

2) La observación de cetáceos ofrece la oportunidad de familiarizarse con temas ambientales y de ser involucradas en esfuerzos a nivel personal, local, regional, nacional e internacional para la conservación del medio ambiente.

3) El desarrollo de programas educativos refuerza las relaciones entre la industria de actividades de observación de cetáceos y las comunidades locales, conectando a la ciudadanía con la comunidad científica.

4) El conocimiento sobre historia natural adquirido gracias a la observación de cetáceos posee un valor intrínseco notable.

5) La actividad de observación de cetáceos ofrece la oportunidad de observar a los animales en la naturaleza, transmitiendo informaciones reales y permitiendo disipar mitos.

6) La observación de cetáceos es un modelo para programas de educación marina a través de viajes de aventura y ecoturismo.

7) La observación de cetáceos ofrece la oportunidad de apreciar y entender la historia, cultura y el medio ambiente local.

Por su parte, el guía (véase figura 6) es el elemento clave de cualquier programa educativo que tenga como finalidad la observación de cetáceos; es la figura que puede potenciar el valor de la experiencia de observación de cetáceos a través de la información ofrecida (Walker et al., 2017). Es él quien tiene la gran capacidad y responsabilidad de transformar un "simple" turista en un "avistador"; de educar y promover cambios individuales en la actitud de los usuarios hacia el medio ambiente (Servidio et al, 2016).

Figura 6: Actividad de guianza en salida turística.

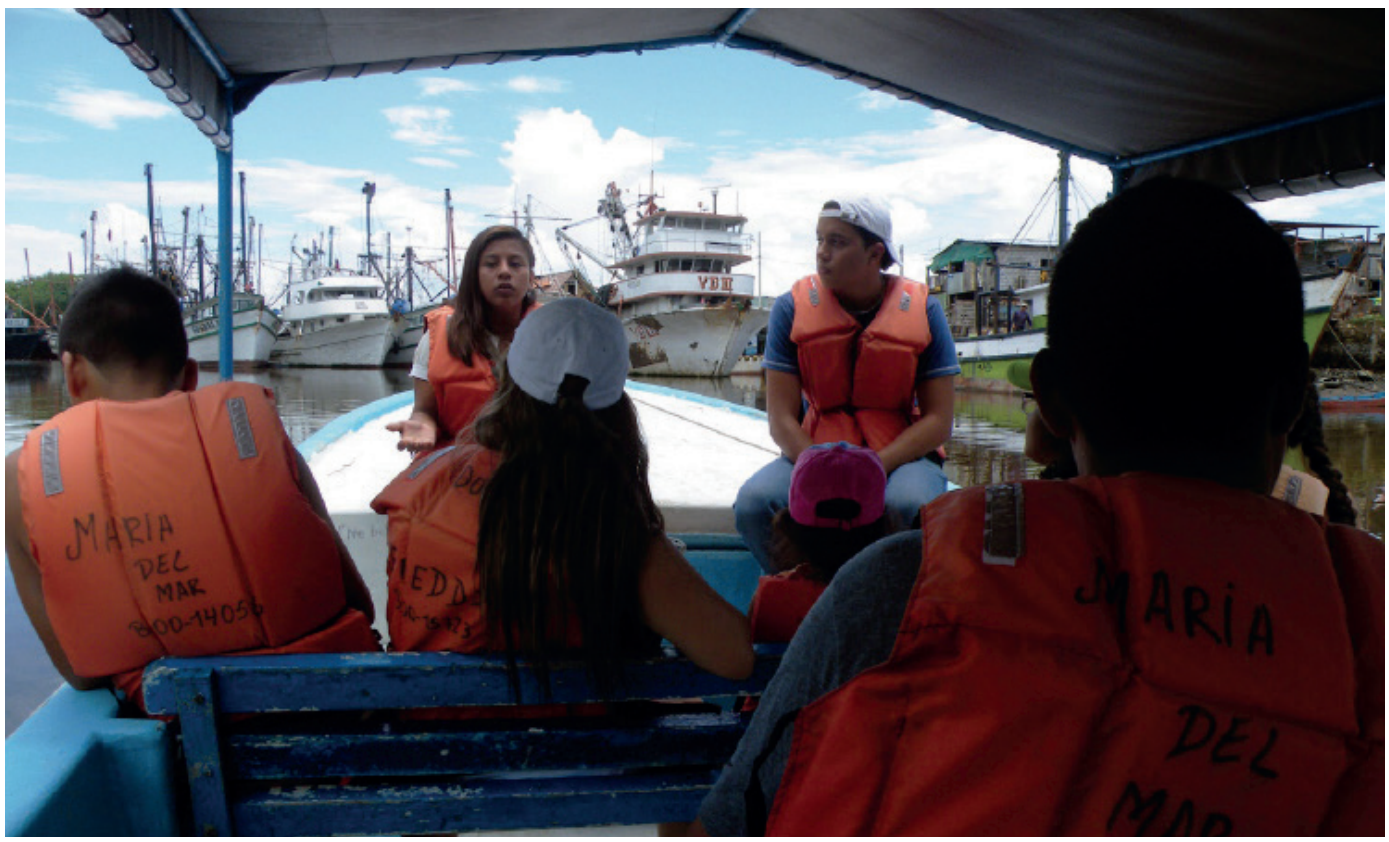

Foto: Rafael Correa. 


\section{Metodología}

Para el estudio de las actividades ecoturísticas de las operadoras de Puerto El Morro que realizan avistamiento de delfines se realizaron 12 acompañamientos a salidas de avistamiento de delfines antes del desarrollo de la guía para el avistamiento de bufeos, en las cuales se evaluó la efectividad en cubrir los aspectos ecológicos considerados de importancia para la realización de guianzas eficientes, y acordes con el principio de ecoturismo (véase figura 7) (The International Ecotourism Society, 2019). La aportación del guía durante el itinerario fue evaluada mediante un formulario de 42 puntos clasificados en siete apartados (adaptaciones de delfín, adaptaciones del manglar, aves, conservación, el delfín-generalidades, el manglar-generalidades, y fisiología del delfín) (véase figura 7). Posteriormente se realizó un curso de $12 \mathrm{~h}$ para los guías ecoturísticos de las operadoras que realizaban salidas en el REVISMEM enfocada en la sostenibilidad, los atractivos faunísticos y los manglares. Aglomerando la información extendida en los cursos, los datos recogidos en las salidas previas al curso y conversaciones con usuarios y operadoras turísticas se determinaron los temas que se encuentran en la guia para el avistamiento de delfines que fue entregada a la comunidad. Con el fin de evaluar las preferencias temáticas respecto a las actividades ecoturísticas se utilizaron dos tipos de cuestionarios aplicados a usuarios de las salidas ecoturísticas en bote. Los cuestionarios utilizados en el proyecto fueron aplicados a adultos de ambos sexos, individualmente, durante la salida turística, con permiso de las operadoras turísticas. Durante nueve salidas se aplicaron 2 cuestionarios a usuarios y se rellenó un formulario de evaluación de la guianza. 22 cuestionarios fueron recolectados con información referente a la motivación que impulso su visita turística buscando, además, registrar cuales fueron los atractivos prioritarios de los turistas a la hora de optar por el paseo en bote en el REVISMEM, y 36 cuestionarios con la información relativa a la apreciación de las normativas de avistamiento (Peña y Leandro, 2006; Pérez, 2013). Mediante los 22 cuestionarios a usuarios referentes a concretar la motivación que les impulso a que realizaran su visita turística se obtuvieron 29 registros de respuesta debido a la libertad de respuesta ofertada y la múltiple respuesta registrada, ya que en ocasiones atribuyeron su visita a más de un atractivo turístico. Al mismo tiempo, se evaluó a los guías sobre temáticas referentes a los atractivos naturales del REVISMEM y también se dio seguimiento a la posterior evolución en calidad de guianza de una guía turística en un lapso de cuatro meses. Esta guia ecoturística participó en una salida evaluada previa participar en el curso ofertado y recibir la información referente a la guía por escrito y tres salidas evaluadas adicionales posteriores. Los resultados fueron analizados mediante $R$ Commander (Fox, Bouchet-Valat, 2019).

Para la elaboración de la guiase utilizó información de fuentes bibliográficas de revistas científicas y de ámbito histórico siempre con relación a los valores naturales encontrados en el área de interés (el REVISMEM), y puntualmente, se nombraron fuentes de medios comerciales que aportan información videográfica seleccionada que es relevante para la sensibilización y refuerzo del guía (Khan, 2018). El apartado dedicado al ecosistema de manglar cubre diferentes especies encontradas en el área protegida del REVISMEM. En el caso de las aves, se reconocieron e investigaron las seis especies más carismáticas y comúnmente divisadas durante las salidas ecoturísticas en el REVISMEM y se incluyeron en la guía entregada a la comunidad para su estudio autónomo. Además del aporte informacional, a lo largo del manuscrito se realizan sugerencias para la mejora de la guianza antes de cada tema, para dirigir la línea argumental durante las actividades ecoturísticas.

Todas las fotografías en este trabajo fueron realizadas durante las salidas de avistamiento de bufeos de Puerto El Morro en el REVISMEM por Eduardo Rafael Correa y por Fernando Félix. Todos los guías de las operadoras turísticas y conductores de bote asociados recibieron la información referida a la "Guía de campo para el avistamiento de delfines en Puerto el Morro (Ecuador).", desarrollada a partir de la investigación y la evaluación de conocimiento de las temáticas referidas a los valores naturales del área.

\section{Resultados y análisis}

Ante la pregunta abierta sobre la razón de la visita a Puerto El Morro y al REVISMEM el 76,2 \% de los usuarios nombraron al delfín como el atractivo que más había influenciado en la decisión. Debido a las respuestas múltiples, la respuesta genérica del turismo o paseo se mencionó como factor influyente en la decisión de visitar el área el 28,6 \% de las ocasiones, mientras que las aves y el manglar fueron mencionadas en el 9,5\% de las ocasiones. Referentemente a la normativa el 100\% de los encuestados expusieron su preferencia a realizar el itinerario en una embarcación que respetara la normativa. 
Además, el 50\% expresaron su aprobación respecto a la aplicación de la normativa por el bienestar de los delfines, el 13,8\% mencionaron la seguridad del pasajero como aspecto de la normativa más relevante, el $27,8 \%$ no dieron razón alguna, mientras que el $5.6 \%$ se limitaron a celebrar la salida. Otro $5.6 \%$ de los encuestados mencionaron respuestas singulares como la importancia de la limpieza o el aprendizaje.

Los resultados evidenciaron la falta de aportaciones de los guías respecto al REVISMEM en áreas temáticas que se consideraron de importancia para la presentación de los atractivos ecoturísticos, tanto la población local de delfines, como en apartado referentes a aves y a ecosistema de manglar (véase figura 7.).

\section{Figura 7: Gráficos mostrando los resultados de los formularios} de evaluación de guianza.

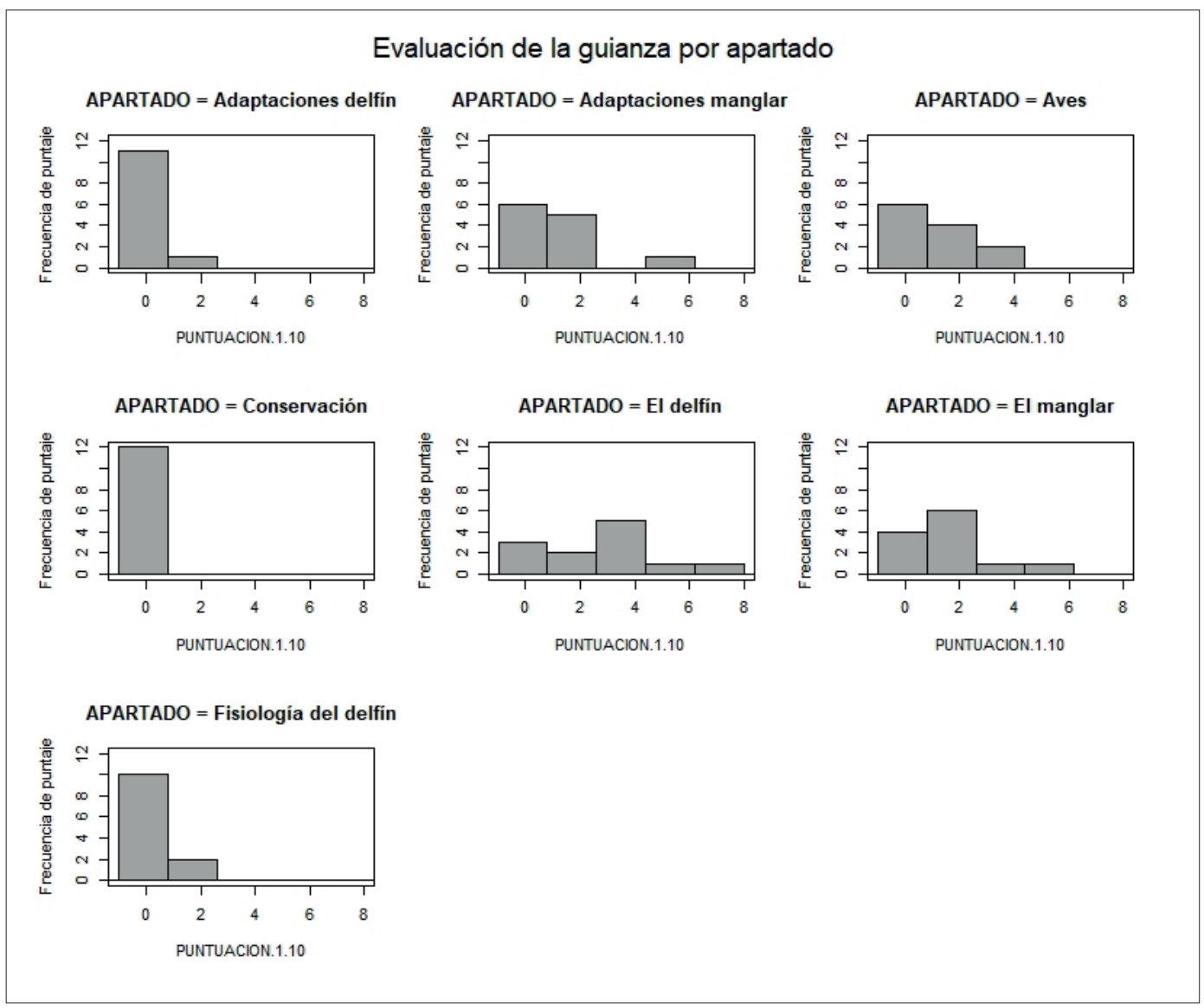

Los datos reflejaron una deficiencia en varios aspectos del conocimiento en la guianza en los temas referentes a cetáceos. Los apartados que menos se desarrollaron fueron los siguientes; los referidos a adaptaciones del delfín y/o su evolución, la fisiología del delfín y la conservación de los valores naturales. $\mathrm{El}$ apartado más desarrollado fue el referente a características generales del delfín.

Los resultados de la evaluación de la guianza durante las actividades de avistamiento de delfines reflejan que los guías, aun con un valor bajo de media $(\bar{x}=2.7)$ y una alta desviación estándar ( $\mathrm{sd}=2.3$ ), enfocaron sus aportes informacionales en los conocimientos generales de los delfines (véase figura 7), pero apenas mencionaron sus adaptaciones $(\overline{\mathrm{x}}=0.21, \mathrm{sd}=0.72)$ y fisiología $(\overline{\mathrm{x}}=0.23, \mathrm{sd}=0.57)$, características que derivan en comportamientos diferenciadores respecto a otras especies. El manglar y sus adaptaciones 
fueron mencionados en segundo orden $(\overline{\mathrm{x}}=1.46, \mathrm{sd}=1.67 \mathrm{y} \overline{\mathrm{x}}=1.57, \mathrm{sd}=1.46)$. Aspectos relevantes a la conservación del ecosistema no fueron mencionados en ninguna ocasión.

Los registros de las guianzas reflejaron una alta diversidad en la información extendida durante las guianzas, lo que indica que existe una improvisación y subjetividad en el resultado de la guianza. Varios apartados resultaron pobremente representados, destacando la evolución del delfín y su fisiología, apartados de gran importancia para la comprensión de la especie y su comportamiento, mientras que la educación ambiental relevante a la conservación de los valores naturales no fue mencionada en ningún momento.

Figura 8: Ejemplo de progresión de resultados favorables en la guianza.

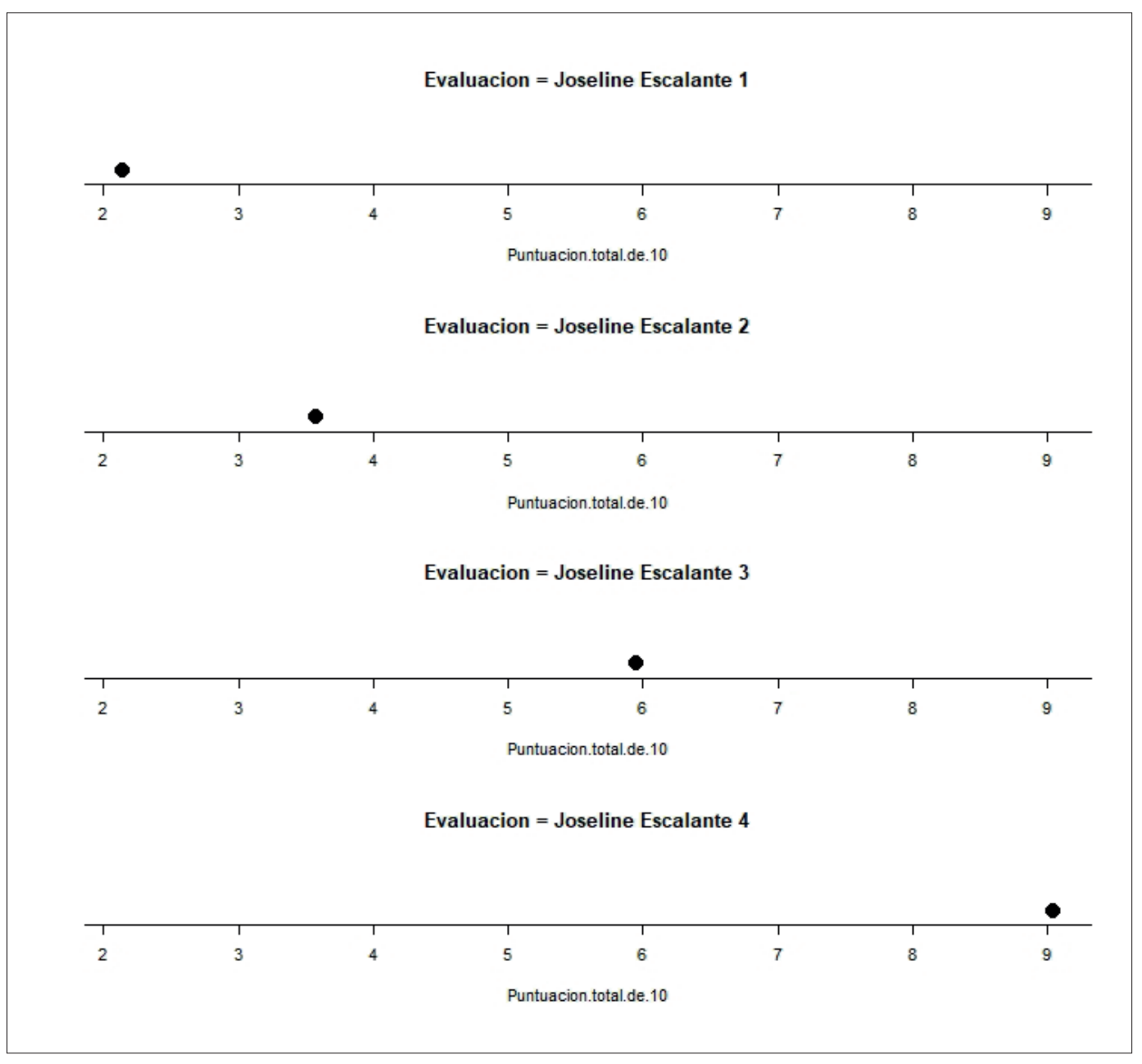

Durante el seguimiento de la guía evaluada se presenció una progresión notable en la evaluación de su actividad de guianza referente a los apartados mencionados anteriormente, incluyendo los relevantes a la conservación de los valores naturales del REVISMEM (véase figura 8). Cabe mencionar que, tras la primera evaluación, se realizó un curso de capacitación y una guianza asesorada, y se brindó la información por escrito de los apartados evaluados para su estudio autónomo que se insertó en la guía otorgada a las operadoras. La progresión de los resultados tras el aporte de la guía se registró desde febrero a julio del 2017, mientras que la primera evaluación se dio en diciembre del 2016. 


\section{Conclusión}

El delfín resultó el atractivo de mayor importancia seguido del turismo o paseo, posicionándose en último lugar las aves y el ecosistema de manglar. La actividad de paseo o turismo podría ser relacionada con el ambiente o paisaje a experimentar durante los trayectos ofrecidos, donde la observación de la naturaleza se podría conectar con el ecosistema de manglar (Pelegrín, 2014).

Gran parte de los usuarios consultados mencionaron su preocupación sobre el estado del delfín y valoraron positivamente la ejecución de las actividades según las normativas, lo cual, puede ser un factor a tener en cuenta para potenciar el respeto de las mismas.

La evaluación de la actividad turística es un recurso que favorece la implementación de actividades a favor de una mejora de la oferta ecoturística, ya que identifica los aspectos que mayor refuerzo requieren. El análisis de la actividad de guianza en términos ecológicos reflejaron las debilidades en el discurso y el conocimiento de áreas temáticas referentes a los delfines, al entorno o ecosistema de manglar, a las aves locales, así como en lo referente a la conservación de estos atractivos naturales, por lo que se concluye que dichos temas deben desarrollarse como un aporte informacional y educativo relevante en el ecoturismo local. Por ello, en la Guía de campo para el avistamiento de delfines se desarrollaron estas temáticas; de forma principal los cetáceos y delfines, y de forma secundaria el ecosistema de manglar y la avifauna, siempre con argumentos que apoyen su conservación. La guía de campo para el avistamiento de delfines en Puerto El Morro (Ecuador) se extiende mayormente en los aspectos referentes específicamente al bufeo o delfín nariz de botella presente en esa área en concreto, ya que, según nuestro estudio y el de Aguirre-Torres (2019), representa el principal atractivo de la reserva. Se debe mencionar que dependiendo del área sobre la cual se trate la guía de campo para el ecoturista el tema predilecto debería variar, tanto según la percepción o la atracción generada en el visitante inicialmente como en los objetivos de conservación que sean prioritarios.

Gracias al análisis de la importancia de los recursos ecoturísticos en términos de popularidad se justificaron los lineamientos más oportunos a la hora de diseñar una guía de campo para los itinerarios ecoturísticos locales. La guía de avistamiento representa un material de apoyo y referencia, para la promoción de un discurso cada vez más desarrollado en la guianza, siempre que exista la prestación a la mejora continua. Es recomendable para un fácil aprendizaje presentar la información en forma de guía rápida, para mayor eficiencia de lectura por el guía, aportando en temáticas referentes los atractivos ecoturísticos más llamativos del área en cuestión y su conservación, en este caso, el REVISMEM.

Se evidenció la progresión de la guía ecoturística evaluada a lo largo del tiempo en posesión del manuscrito con la información ofrecida (véase figura 8), además, la guía favorece no solo el aprendizaje autónomo, sino que también busca la motivación a la hora de realizar la actividad de guianza, representando un material didáctico que, a la vez, valora el ejercicio del guía debido a su directa implicación con la sensibilización del público visitante y la conservación de la naturaleza.

El conocimiento de las especies silvestres y su entorno es el primer paso para una protección de los valores naturales y las guías, promueven el acercamiento continuo al valor natural y su conservación. El conocimiento de las especies, y en concreto, el conocimiento de la especie de mayor poder de atracción para el turista en el área, el delfín, genera una mayor capacidad de comprensión respecto a la urgencia de protección de la especie y el estado de su población local. En el REVISMEM se encuentran especies interesantes que el guía ecoturístico es capaz de acercar al público en general, apoyándose en información contrastada dirigida a completar los objetivos educacionales de la actividad ecoturística. Las aves y el ecosistema de manglar son atractivos que, junto al delfín, ofrecen una experiencia única que se debe respetar y conservar. Esta población de delfines tan reducida es tan interesante y atractiva como frágil, por lo que su conservación resulta de gran importancia.

\section{Biliografia}

Aguirre-Torres, G. (2019). El turismo sostenible comunitario en Puerto el Morro: análisis de su aplicación e incidencia económica. Revista Universidad y Sociedad, 11(1), 289-294.

Akoglu, E., Salihoglu, B., Libralato, S., Oguz, T., y Solidoro, C. (2014). An indicator-based evaluation of Black Sea food web dynamics during 1960-2000. Journal of Marine Systems, 134, 113-125.

Allen, M. C., y Read, A. J. (2000). Habitat selection of foraging bottlenose dolphins in relation to boat density near Clearwater, Florida. Marine Mammal Science, 16(4), 815-824.

Alpers, A. (1961). Dolphins: the myth and the mammal. London: J. Murray. 
Ballance, L. T. (2016). Cetacean ecology. Lat. Am. J. Aquat. Mamm, 2, 87-94.

Baum, J. K., y Worm, B. (2009). Cascading top down effects of changing oceanic predator abundances. Journal of Animal Ecology, 78(4), 699-714.

Bekoff, M. (2004). Wild justice and fair play: Cooperation, forgiveness, and morality in animals. Biology and Philosophy, 19(4), 489-520.

BBC. (2012). Dolphins deserve same rights as humans, say scientists. Recuperado de: http://www.bbc. com/news/world-17116882

Casini, M., Lövgren, J., Hjelm, J., Cardinale, M., Molinero, J. C., y Kornilovs, G. (2008). Multi-level trophic cascades in a heavily exploited open marine ecosystem. Proceedings of the Royal Society of London B: Biological Sciences, 275(1644), 1793- 1801.

Carlson, C. (2012). A Review of Whale Watch Guidelines and Regulations Around the World: Version 2012. International Whaling Commission.

IFAW. (1997). Report of the workshop on the educational value of whale watching. 8-11 may of 1997. Provincetown, Massachusetts, USA: International Fund for Animal Welfare. Recuperado de https:// whalesenseblog.files.wordpress.com/2013/03/report-of-the-educational-value-of-whale-watching.pdf [Consultado el 25/02/2020]

Culloch, R. M., Anderwald, P., Brandecker, A., Haberlin, D., McGovern, B., Pinfield, R., ... y Cronin, M. (2016). Effect of construction-related activities and vessel traffic on marine mammals. Marine Ecology Progress Series, 549, 231-242.

Currey, R., Boren, L. J., Sharp, B. R., y Peterson, D. B. (2012). A risk assessment of threats to Maui's dolphins (p. 55). New Zealand Ministry for Primary Industries and Department of Conservation.

Daniel, T. C., Muhar, A., Arnberger, A., Aznar, O., Boyd, J. W., Chan, K. M., ... y Grêt-Daskalov, G. M. (2002). Overfishing drives a trophic cascade in the Black Sea. Marine Ecology Progress Series, 225, 53-63.

Daskalov, G. M., Grishin, A. N., Rodionov, S., y Mihneva, V. (2007). Trophic cascades triggered by overfishing reveal possible mechanisms of ecosystem regime shifts. Proceedings of the National Academy of Sciences, 104(25), 10518-10523.

Deyermond, A. (2004). La tradición de los bestiarios en la antigua lírica popular hispánica. In De la canción de amor medieval a las soleares: profesor Manuel Alvar "In memoriam". (Actas del Congreso Internacional 'Lyra minima oral III', Sevilla, 87-100.

Dill, L., Heithaus, M., y Walters, C. (2003). Behaviorally Mediated Indirect Interactions in Marine Communities and Their Conservation Implications. Ecology, 84(5), 1151-1157.

Félix, F. (2013). Los bufeos del golfo de Guayaquil, guía de campo. www.museodeballenas.org. Accedido a la página web el 3 de abril de 2017.

Forestell, P. H. (2007). Protecting the ocean by regulating whale watching: The sound of one hand clapping. Marine Wildlife and Tourism Management: Insights from the Natural and Social Sciences. CABI Publishing, Oxfordshire, UK, 272-293.

Fox J, Bouchet-Valat M. (2019). Rcmdr: R Commander. R package version 2.6-0, http://socserv.socsci. mcmaster.ca/jfox/Misc/Rcmdr/.

Frank, K. T., Petrie, B., Choi, J. S., y Leggett, W. C. (2005). Trophic cascades in a formerly cod-dominated ecosystem. Science, 308(5728), 1621-1623.

García-Cegarra, A. M., y Pacheco, A. S. (2017). Whale-watching trips in Peru lead to increases in tourist knowledge, pro-conservation intentions and tourist concern for the impacts of whale-watching on humpback whales. Aquatic Conservation: Marine and Freshwater Ecosystems.

Grimm D. y Miller G. (2010). American American Association for the Advancement of Science (AAAS). De http://www.sciencemag.org/news/2010/02/dolphin-person

Hastie, G. D., Wilson, B., Tufft, L. H., y Thompson, P. M. (2003). Bottlenose dolphins increase breathing synchrony in response to boat traffic. Marine Mammal Science, 19(1), 74.

Hicks, E. L. (1887). Iasos. The Journal of Hellenic Studies, 8, 83-118.

Hoyt, E., y Iñíguez, M. (2008). The state of whale watching in Latin America. WDCS, Chippenham, UK.

Janik, V. M., y Thompson, P. M. (1996). Changes in surfacing patterns of bottlenose dolphins in response to boat traffic. Marine mammal science, 12(4), 597-602.

Jiménez, P. J., y Álava, J. J. (2015). Strand-feeding by coastal bottlenose dolphins (Tursiops truncatus) in the Gulf of Guayaquil, Ecuador. Latin American Journal of Aquatic Mammals, 10(1), 33-37.

Khan, D. (2018). Sustainable learning a new paradigm in environmental education: a study to facilitate awareness among the school children in some selected schools in Nadia district, West Bengal, India. International Research Journal of Multidisciplinary studies, 4(3). 
Marten, K., y Psarakos, S. (1994). Evidence of self-awareness in the bottlenose dolphin (Tursiops truncatus).

Mauri, M. M. (2004). El Mar kuna. Representación y uso de los recursos marinos en Kuna Yala (Panamá). Perifèria: revista de recerca i formació en antropologia, (1).

Ministerio de Turismo de Ecuador (2014). Acuerdo Ministerial 4. Registro Oficial 278, de 30 de junio del 2014, normativa que regula la observación de ballenas y delfines en ecuador. Recuperado de http:// www.turismo.gob.ec/wp-content/ uploads/2016/04/NORMATIVA-QUE-REGULA-OBSERVACION-DEBALLENAS-Y-DELFINES-EN-ECUADOR.pdf [Consultado el 11/08/2020]

Nakahara, F. (1999). Influences of the underwater man-made noise on acoustic behavior of dolphins. otsucht marine scjence 24, IS.23.

Nowacek, S. M., Wells, R. S., y Solow, A. R. (2001). Short-term effects of boat traffic on bottlenose dolphins, Tursiops truncatus, in Sarasota Bay, Florida. Marine Mammal Science, 17(4), 673-688.

Pelegrín, G. A. B. (2014). El turismo de naturaleza en espacios naturales. El caso del Parque Regional de las Salinas y Arenales de San Pedro del Pinatar. Cuadernos de turismo, (34), 33-51.

Pérez, J. I. J. (2013). Manejo de recursos naturales y procesos agrícolas para el turismo rural campesino en un Ejido de transición ecológica de México. (Primera parte). PASOS. Revista de Turismo y Patrimonio Cultural, 11(2), 327-342.

Peña, A., y Leandro, R. A. L. (2006). Opinión del sector empleador público sobre el desempeño laboral del Licenciado en Turismo egresado de la Universidad de Oriente, Núcleo Nueva Esparta. PASOS. Revista de Turismo y Patrimonio Cultural, 4(2), 235-254.

Remage-Healey, L., Nowacek, D. P., y Bass, A. H. (2006). Dolphin foraging sounds suppress calling and elevate stress hormone levels in a prey species, the Gulf toadfish. Journal of Experimental Biology, 209(22), 4444-4451.

Reiss, D., y Marino, L. (2001). Mirror self-recognition in the bottlenose dolphin: A case of cognitive convergence. Proceedings of the National Academy of Sciences, 98(10), 5937-5942.

Servidio, A., Elejabeitia, C., Lopez, T., y Iani, V. (2016). La figura del guía en la industria de observación de cetáceos. Gobierno de Canarias. Consejería de política territorial y medio ambiente. Sociedad Española de Cetáceos.

Sistema Nacional de Información (2015). Plan de Desarrollo y Ordenamiento Territorial del GAD Parroquial de El Morro 2015-2019. Secretaria Nacional de Planificación y Desarrollo. Recuperado de http://app.sni. gob.ec/sni-link/sni/PORTAL_SNI/data_sigad_plus/sigadplusdocumentofinal/0968538740001_PLAN\%20 DE\%20DESARROLLO\%20Y\%20ORDENAMIENTO\%20TERRITORIAL\%20RURAL\%20DEL\%20 MORRO\%2028\%2010\%202015\%20(1)_30-10-2015_16-38-46.pdf [Consultado el 11/08/2020)]

Spalding, M., y Blumenfeld, J. (1997, November). Legal aspects of whale watching in North America. In Simposio Legal Aspects for Whalewatching. Punta Arenas-Chile (pp. 17-20).

Stensland, E., Carlen, I., Särnblad, A., Bignert, A., y Berggren, P. (2006). Population size, distribution, and behavior of indo pacific bottlenose (Tursiops aduncus) and humpback (Sousa chinensis) dolphins off the south coast of Zanzibar. Marine mammal science, 22(3), 667-682.

Tejedor Calvo, S. (2016). Yunka Wasi: historias que cuenta la selva. Editorial UOC.

Traina, G. (2005). Notes on Hellenism in the Iranian East (Classico-Oriental Notes, 6-8). Iran y the Caucasus, 1-14.

Walker, K., y Weiler, B. (2017). A new model for guide training and transformative outcomes: a case study in sustainable marine-wildlife ecotourism. Journal of Ecotourism, 16(3), 269-290.

Wilson, B., y Dill, L. M. (2002). Pacific herring respond to simulated odontocete echolocation sounds. Canadian Journal of Fisheries and Aquatic Sciences, 59(3), 542-553. 